\title{
Quasi-static and dynamic hardening and fracture strain of A2-70 stainless steel under different temperatures
}

\author{
Giuseppe Mirone $^{1}$ and Raffaele Barbagallo ${ }^{1}$ \\ ${ }^{1}$ University of Catania
}

May 5, 2020

\begin{abstract}
In this paper, the behaviour of A2-70 stainless steel is investigated by performing tensile tests on round specimens at different temperatures under quasi-static and dynamic conditions. The relationship between thermal softening and strain is firstly investigated, highlighting that the variability of the necking strain under different temperatures is a proof of such interaction. The competing effects of strain rate and temperature in respectively delaying and anticipating the necking onset are also quantified analytically, referring to multiplicative hardening models with and without the coupling of strain and temperature. Then, the comparison of necking strains from static and dynamic (Hopkinson bar) tests at different temperatures is analysed, for understanding which effect among thermal softening and dynamic stress amplification prevails in anticipating/delaying the necking. Fracture strains and the shapes of specimens at failure are finally related to the respective strain rates and temperatures.
\end{abstract}

Quasi-static and dynamic hardening and fracture strain of A2-70 stainless steel under different temperatures Giuseppe Mirone11* Corresponding author. Tel.: +39 095738 2418.E-mail address: gmirone@dii.unict.it, Raffaele Barbagallo

University of Catania, DICAR - Department of Civil Engineering and Architecture, Via Santa Sofia 64 95125 - Catania, Italy

Abstract

In this paper, the behaviour of A2-70 stainless steel is investigated by performing tensile tests on round specimens at different temperatures under quasi-static and dynamic conditions. The relationship between thermal softening and strain is firstly investigated, highlighting that the variability of the necking strain under different temperatures is a proof of such interaction. The competing effects of strain rate and temperature in respectively delaying and anticipating the necking onset are also quantified analytically, referring to multiplicative hardening models with and without the coupling of strain and temperature. Then, the comparison of necking strains from static and dynamic (Hopkinson bar) tests at different temperatures is analysed, for understanding which effect among thermal softening and dynamic stress amplification prevails in anticipating/delaying the necking. Fracture strains and the shapes of specimens at failure are finally related to the respective strain rates and temperatures.

Keywords: Necking; Tensile instability; Steel; Temperature; Strain Rate; Thermal Softening; Fracture.

\begin{tabular}{llll}
\hline Nomenclature & Nomenclature & Nomenclature & Nomenclature \\
\hline$d$ & Diameter of the specimen & $\varepsilon_{N-D}$ & Dynamic necking onset true strain \\
$d_{0}$ & Initial diameter of the specimen & $\varepsilon_{N-D T}$ & Dynamic necking onset true strain \\
$F$ & Force acting on the specimen & $\varepsilon_{N-S}$ & Necking onset true strain in quasi-
\end{tabular}




\begin{tabular}{llll}
\hline Nomenclature & Nomenclature & Nomenclature & Nomenclature \\
\hline$K, n$ & Hollomon equation parameters & $\varepsilon_{N-T}$ & Necking onset true strain in quasi- \\
$R$ & Strain rate amplification of the equivalent stress & $\varepsilon_{\text {true }}$ & True strain \\
$S$ & Thermal softening & $\dot{\varepsilon}_{\text {true }}$ & True strain rate \\
$T$ & Temperature & $\sigma_{\mathrm{Eq}}$ & Equivalent stress \\
& & $\sigma_{E q-S}$ & Equivalent stress in a static test \\
& & $\sigma_{\text {true }}$ & True stress \\
& $\sigma_{\text {yield }}$ & Yield stress \\
\hline
\end{tabular}

\section{Introduction}

The combined effects of strain, strain rate and temperature on the behaviour of metals have been widely studied in the literature. Ruggiero et al. analysed the ductility variations of an ADI JS/1050-6 iron due to different strain rate and temperatures ${ }^{1}$, Scapin et al. made similar investigations on pure copper ${ }^{2}$ while Sasso et al. ${ }^{3}$ studied the strain rate sensitivity of AA7075 aluminum alloy at different initial temper states. Mirone et al. ${ }^{4}$ and Mirone et al. ${ }^{5}$ highlighted the interactions between the necking onset, the true strain rate and the effective dynamic amplification of the material equivalent stress-strain curve in different metals.

Hart $^{6}$ and Ghosh ${ }^{7}$ evaluated the necking onset strain at high strain rates obtaining different mathematical relationships due to different starting hypothesis about the elongation conditions of the specimen. A modified version of the Hart criterion was proposed by Guan ${ }^{8}$ while $\operatorname{Lin}^{9}$ obtained the instability conditions for uniaxial tension specimens of materials characterized by a significant strain rate sensitivity. Hart, Ghosh and Lin approaches do not include the grow of the temperature inevitably occurring during high strain rate deformations due to the adiabatic development of plastic work inside the material. The plastic work during a test is proportional to the area subtended by the equivalent stress-strain curve of the material. Then, the heat effectively produced is proportional to such plastic work, through the Taylor-Quinney coefficient. Kapoor \& Nemat-Nasser ${ }^{10}$ and Walley et al. ${ }^{11}$ obtained that almost all the plastic work developed during high strain rate tests of different metals is converted to heat while Jovic et al. ${ }^{12}$ and Rittel et al. ${ }^{13}$ calculated that only lower fractions of it are converted to heat.

The equivalent stress-strain curve of the material, necessary to correctly evaluate the plastic work and the temperature, can be obtained from the true curve using the well-known procedure proposed by Bridgman ${ }^{14}$. An alternative procedure, simpler and more accurate, was proposed by Mirone ${ }^{15}$. For cylindrical specimens, it is simple to obtain the starting true curve by monitoring the diameter of the minimum cross-section of the specimen. Instead, for thick rectangular specimens, the true curve extrapolation is not so straightforward. To address such issue, Mirone et al. ${ }^{16}$ recently proposed a procedure to obtain the true curve of thick flat specimens at locally necked material points, starting from the global engineering variables, i.e. experimental force and elongation of the gage length.

Other methods allow to indirectly obtain the equivalent curve without the necessity of calculating the true curve. Peroni et al. ${ }^{17}$ proposed an equivalent curve extrapolation procedure based on the monitoring of the entire neck profile; the inverse method converges when the calibrated material model is able to reproduce such profile.

Sasso et al. ${ }^{18}$ proposed an analytical method, alternative to the classical inverse FEM-based procedure, which gave results in good agreement with those obtained through finite element simulations, with a good matching to experimental data.

In this paper, the behavior of A2-70 stainless steel at different temperatures and under quasi-static and dynamic conditions is investigated; in particular, the combined effects of strain, strain rate and temperature on the necking onset have been analyzed both analytically and experimentally, giving some new insights about the complex interaction between such variables.

Quasi-static and dynamic instability conditions 
The strain for tensile instability onset can be obtained through the well-known Considère mathematical condition, taking into account also the eventual effects of true strain rate $\dot{\varepsilon}_{\text {True }}$ and temperature $T$ together with their variations. In fact, during a general dynamic test, materials are typically subjected to strain rate increase due to finite rise times of the loads and to temperature increase due to the adiabatic conversion of plastic work into heat. The Considère condition is shown in eq. (1) where $\sigma_{\mathrm{Eq}}, \varepsilon_{\text {True }}$ and $\varepsilon_{N-\mathrm{DT}}$ are respectively the equivalent stress, the true strain and the necking strain affected by both dynamics and temperature effects.

$\sigma_{\mathrm{Eq}}-\frac{d \sigma_{\mathrm{Eq}}\left(\varepsilon_{\text {True }}, \dot{\varepsilon}_{\text {True }}, T\right)}{d \varepsilon_{\text {True }}}=\sigma_{\mathrm{Eq}}-\frac{\partial \sigma_{\mathrm{Eq}}}{\partial \varepsilon_{\text {True }}}-\frac{\partial \sigma_{\mathrm{Eq}}}{\partial \dot{\varepsilon}_{\text {True }}} \bullet \frac{\partial \dot{\varepsilon}_{\text {True }}}{\partial \varepsilon_{\text {True }}}-\frac{\partial \sigma_{\mathrm{Eq}}}{\partial T} \bullet \frac{\partial T}{\partial \varepsilon_{\text {True }}}=0 \rightarrow \varepsilon_{N-D T}$

Here a general simple multiplicative material model is assumed, in which the equivalent stress $\sigma_{\mathrm{Eq}}$ is the product of the equivalent stress under quasi-static conditions and room temperature, $\sigma_{E q-S}$, by the strain rate amplification function $R$ and by the thermal softening function $S$, as shown in eq. (2).

$\sigma_{\text {Eq }}\left(\varepsilon_{\text {True }}, \dot{\varepsilon}_{\text {True }}, T\right)=\sigma_{E q-S}\left(\varepsilon_{\text {True }}\right) \bullet R\left(\dot{\varepsilon}_{\text {True }}\right) \bullet S(T)(2)$

The complete uncoupling between the three relevant variables $\varepsilon_{\text {True }}, \dot{\varepsilon}_{\text {True }}$ and $T$, is tentatively assumed now, meaning that each function is supposed to only depend on its relevant variable and to be independent of the remaining two variables.

For simplifying the comparative evaluation of strain rate and temperature effects on the necking strain, the quasi-static flow curve at room temperature is assumed here to follow the Hollomon relationship $\sigma_{E q-S}=$ $K \bullet \varepsilon_{\text {True }^{n}}$. In such reference condition (static rate and room temperature), the necking strain is equal to the hardening exponent $\varepsilon_{N}=n$.

Eq. (2) introduced within eq. (1) yields to eq. (3).

$\sigma_{E q-S} \bullet R \bullet S-\frac{\partial \sigma_{E q-S}}{\partial \varepsilon_{\text {True }}} \bullet R \bullet S-\sigma_{E q-S} \bullet S \bullet \frac{\partial R}{\partial \dot{\varepsilon}_{\text {True }}} \bullet \frac{\partial \dot{\varepsilon}_{\text {True }}}{\partial \varepsilon_{\text {True }}}-\sigma_{E q-S} \bullet R \bullet \frac{\partial S}{\partial T} \bullet \frac{\partial T}{\partial \varepsilon_{\text {True }}}=0 \rightarrow \varepsilon_{N-D T}$

Given the above framework, it is possible to analyze separately the effects of strain rate and of temperature on the necking inception.

For assessing the effect of strain rate alone on the necking onset, we can refer to an dynamic test under ideal isothermal conditions at room temperature (e.g. according to the multiple step procedure by Ashuach et al. ${ }^{19}$ ), so that $\frac{\partial T}{\partial \varepsilon_{\text {True }}}=0$ and $S=1$ : the uncoupling in eq. (2) together with the dynamic isothermal condition deliver the necking strain $\varepsilon_{N-D}$ in eq. (4).

$\varepsilon_{N-D}=\frac{n}{1-\frac{1}{R} \cdot \frac{\partial R}{\partial \dot{\varepsilon} \text { True }} \cdot \frac{\partial \dot{\varepsilon} \text { True }}{\partial \varepsilon_{\text {True }}}}(4)$

To understand the influence of the strain rate effect on the necking inception we have to compare the necking strain of eq. (4) with the quasi-static necking strain that is equal to $n$, i.e. we must evaluate whether the denominator of the ratio in eq. (4) is lower or higher than one.

In a standard direct-tension split Hopkinson tension bar (SHTB) test, true strain rate typically increases with strain during the rise time of the loading wave, for only becoming constant during the final plateau phase, until necking onset. Moreover, the strain rate amplification factor $R$ of most metals is typically positive and increasing with respect to strain rate. Therefore, all the factors in the denominator of eq. (4) are positive, i.e. $\varepsilon_{N-D}>\varepsilon_{N}$.

It is worth noting that if the strain rate is constant, then $\frac{\partial \dot{\varepsilon}_{\text {True }}}{\partial \varepsilon_{\text {True }}}=0$ and eq. (4) predicts that $\varepsilon_{N-D}=n$ : according to the assumptions made so far (uncoupled multiplicative hardening), only variable strain rates can affect the necking by delaying its onset.

Then, for assessing the effect of the temperature alone on the necking inception, we assume constant quasistatic strain rate so that $\frac{\partial \dot{\varepsilon}_{\text {True }}}{\partial \varepsilon_{\text {True }}}=0$ and $R=1$ : this condition can be easily implemented by static experiments under controlled temperature and the resulting necking strain is given by eq. (5).

$\varepsilon_{N-T}=\frac{n}{1-\frac{1}{S} \cdot \frac{\partial S}{\partial T} \cdot \frac{\partial T}{\partial \varepsilon \text { True }}}(5)$ 
In real SHTB tests the temperature always increases with strain. Moreover the thermal softening $S$ is typically positive and decreases with temperature. Therefore, the denominator of eq. (5) is always greater than one and, consequently, $\varepsilon_{N-T}<\varepsilon_{N-S}$.

Again it is worth noting that if the temperature is constant, then $\frac{\partial T}{\partial \varepsilon_{\text {True }}}=0$ and eq. (5) predicts that $\varepsilon_{N-T}=n$ : the uncoupled hardening assumed so far implies that only variable temperatures can affect the necking by anticipating its onset.

Summarizing, from eq. (4) and eq. (5) it is possible to see that, if the uncoupling of eq. (2) is really taking place, constant strain rate or temperature should not change the necking initiation strain in comparison to the quasi-static case at room temperature. At the same time it is understood that, in standard SHTB tests, two opposite mechanisms, caused by the variation of temperature and strain rate, compete in respectively anticipating and delaying the necking inception and it is not possible, a priori, to know which one prevails.

1. Experimental Tests on A2-70 steel cylindrical specimens

2. Experimental procedures

An experimental campaign on A2-70 steel specimens, including quasi-static tensile tests by motor driven machines at different temperatures (room temperature, $80{ }^{\circ} \mathrm{C}, 140{ }^{\circ} \mathrm{C}, 200{ }^{\circ} \mathrm{C}, 300{ }^{\circ} \mathrm{C}$ ) and dynamic tensile tests by SHTB at room temperature with incident waves of 15 and $26 \mathrm{kN}$, has been carried out. All the tests have been conducted on nominally identical specimens, with a minimum cross section diameter of $3 \mathrm{~mm}$ and a gage length of $9 \mathrm{~mm}$. The details of the campaign are shown in Table 1 where the reference true strain rate is the true strain rate reached before the necking onset. Such value has been chosen as representative for the entire test considering that true strain rate varies greatly during a dynamic tests. The obtained results have been analyzed with particular attention to the necking phenomenon in order to evaluate the influences of temperature and strain rate on its onset.

In all the tests, the minimum cross-section diameter is optically measured during the entire test thanks to standard video camera in the quasi-static tests and high frame rate camera in the dynamic ones. From such data it was possible to calculate the true stress, the true strain and the true strain rate as shown in eqs. (6), (7) and (8).

$$
\begin{aligned}
& \sigma_{\text {True }}=\frac{F}{\pi / 4 \bullet d^{2}}(6) \\
& \varepsilon_{\text {True }}=2 \bullet \operatorname{Ln}\left(\frac{d_{0}}{d}\right)(7) \\
& \dot{\varepsilon}_{\text {True }}=\frac{\partial \varepsilon_{\text {True }}(t)}{\partial t}(8)
\end{aligned}
$$

\begin{tabular}{|c|c|c|c|}
\hline Test Series & Test Name & Reference True Strain Rate $\left[\mathrm{s}^{-1}\right]$ & Test Environment Temperature $\left[{ }^{\circ} \mathrm{C}\right]$ \\
\hline \multirow{3}{*}{$\overline{\text { Static } \mathrm{T}_{\mathrm{ROOM}}}$} & $\mathrm{S}-\mathrm{T}_{\mathrm{ROOM}}-01$ & 0.003 & 19 \\
\hline & $\mathrm{S}-\mathrm{T}_{\mathrm{ROOM}}-02$ & 0.003 & 22 \\
\hline & S-T $\mathrm{TROM}-03$ & 0.003 & 22 \\
\hline \multirow[t]{2}{*}{ Static T80 } & S-T80-01 & 0.003 & 80 \\
\hline & S-T80-02 & 0.003 & 80 \\
\hline \multirow[t]{2}{*}{ Static T140 } & S-T140-01 & 0.003 & 140 \\
\hline & S-T140-02 & 0.003 & 140 \\
\hline \multirow[t]{2}{*}{ Static T200 } & S-T200-01 & 0.003 & 200 \\
\hline & S-T200-02 & 0.003 & 200 \\
\hline \multirow[t]{3}{*}{ Static T300 } & S-T300-01 & 0.003 & 300 \\
\hline & S-T300-02 & 0.003 & 300 \\
\hline & S-T300-03 & 0.003 & 300 \\
\hline \multirow[t]{2}{*}{ Dynamic $\mathrm{T}_{\mathrm{ROOM}}$} & $\mathrm{D}-01(15 \mathrm{kN})$ & 700 & 20 \\
\hline & $\mathrm{D}-02(15 \mathrm{kN})$ & 890 & 20 \\
\hline
\end{tabular}

Table 1. Summary of the A2-70 Experimental Campaign 


\begin{tabular}{llll}
\hline Test Series & Test Name & Reference True Strain Rate $\left[\mathbf{s}^{\mathbf{- 1}}\right]$ & Test Environment Temperature $\left[{ }^{\circ} \mathbf{C}\right]$ \\
\hline & D-03 $(26 \mathrm{kN})$ & 1800 & 20 \\
& D-04 $(26 \mathrm{kN})$ & 1850 & 20 \\
\hline
\end{tabular}

Analysis of the necking inception in the quasi-static tests at different temperatures

In order to assess the influence of the thermal softening on the necking inception, the quasi-static curves at different temperatures have been analysed. The obtained quasi-static experimental true curves are shown in Fig. 1 while the mean yield stress and necking strains, obtained for each group of tests by the maximum load condition, are reported in Table 2.

Such results clearly demonstrate that despite the temperature is maintained constant during each test, it has a great effect in reducing the necking inception strain, the latter varying from 0.44 at room temperature to 0.18 at $300{ }^{\circ} \mathrm{C}$. This is in contrast with eq. (5), predicting that whatever temperature, if constant, cannot affect the necking onset.

Such fact means that the thermal softening cannot only depend on the temperature as postulated in eq. (5), but it must also include a direct dependence on the strain.

In other words, different constant temperatures do not simply scale the quasi-static flow curves by constant factors leaving them homothetic to each other, but such different temperatures also change the shapes of the stress-strain curves during the straining history thanks to a certain degree of strain-dependency; otherwise the necking inception strain would not have changed.

Table 2. Quasi-static Yield Stress and Necking Strain at different temperatures

\begin{tabular}{|c|c|c|c|c|c|}
\hline & S-T $T_{\text {ROOM }}(20)$ & S-T80 & S-T140 & S-T200 & S-T300 \\
\hline$\sigma_{\text {yield }}[M P a]$ & 447.5 & 460 & 420 & 420 & 400 \\
\hline$\varepsilon_{N-T}$ & 0.44 & 0.35 & 0.19 & 0.18 & 0.18 \\
\hline
\end{tabular}

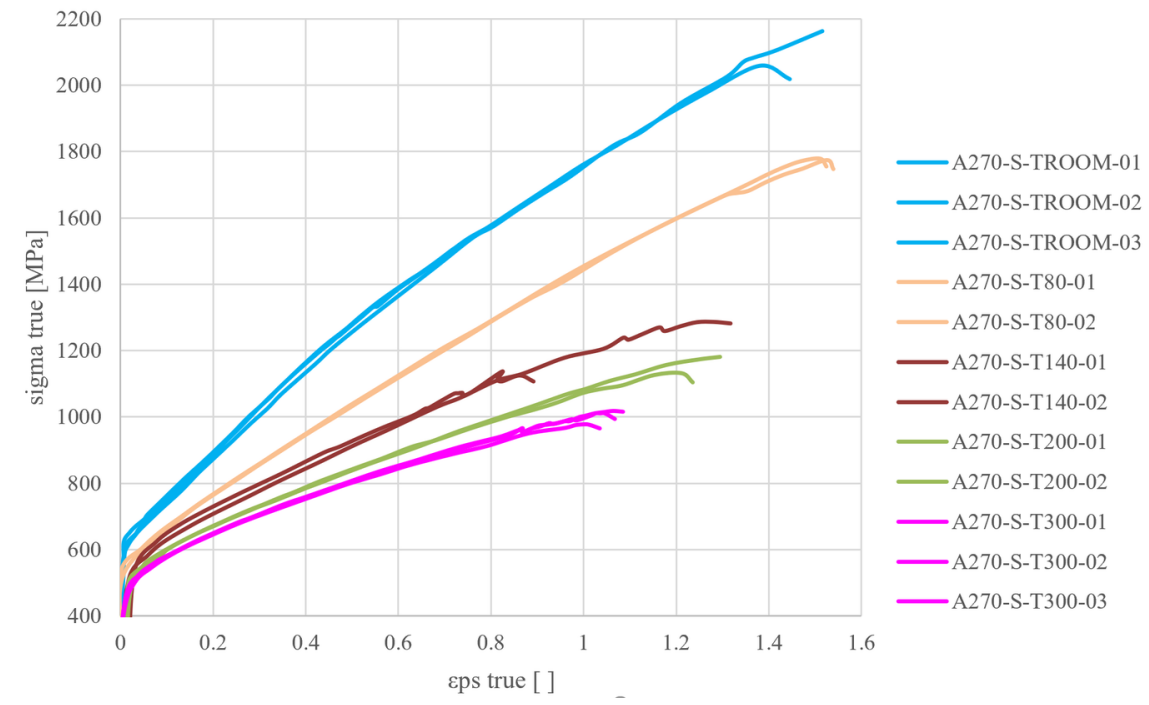

Fig. 1. True stress-true strain experimental data of the quasi-static tests at different temperature

Then, still assuming a multiplicative hardening function like eq. (2) but now discarding any assumption 
about uncoupling of variables, the experimental values of the thermal softening are derived at different instants of the tests by calculating $S=\sigma_{\mathrm{Eq}}\left(\varepsilon_{\text {True }}, T\right) / \sigma_{\mathrm{Eq}}\left(\varepsilon_{\text {True }}, T_{\mathrm{Room}}\right)$. The resulting softening values are then related to the corresponding current values of strain and temperature, so that a general two-variables function $S(T, \varepsilon)$ is then derived as a best-fit of such experimental data associated in triplets of the kind $T, \varepsilon, S$.

The equivalent stress-strain functions at all temperatures, for calculating the above softening values, are derived from each experiment by correcting the respective true curve through the MLR function ${ }^{15}$.

The bestfit function $\mathrm{S}(T, \varepsilon)$ is plotted in Fig. 2 and clearly shows a remarkable dependence of $S$ on both $T$ and $\varepsilon$. The coupling of temperature and strain is frequently neglected in the literature, but for materials like the A2-70 steel it is clear that it cannot be neglected.

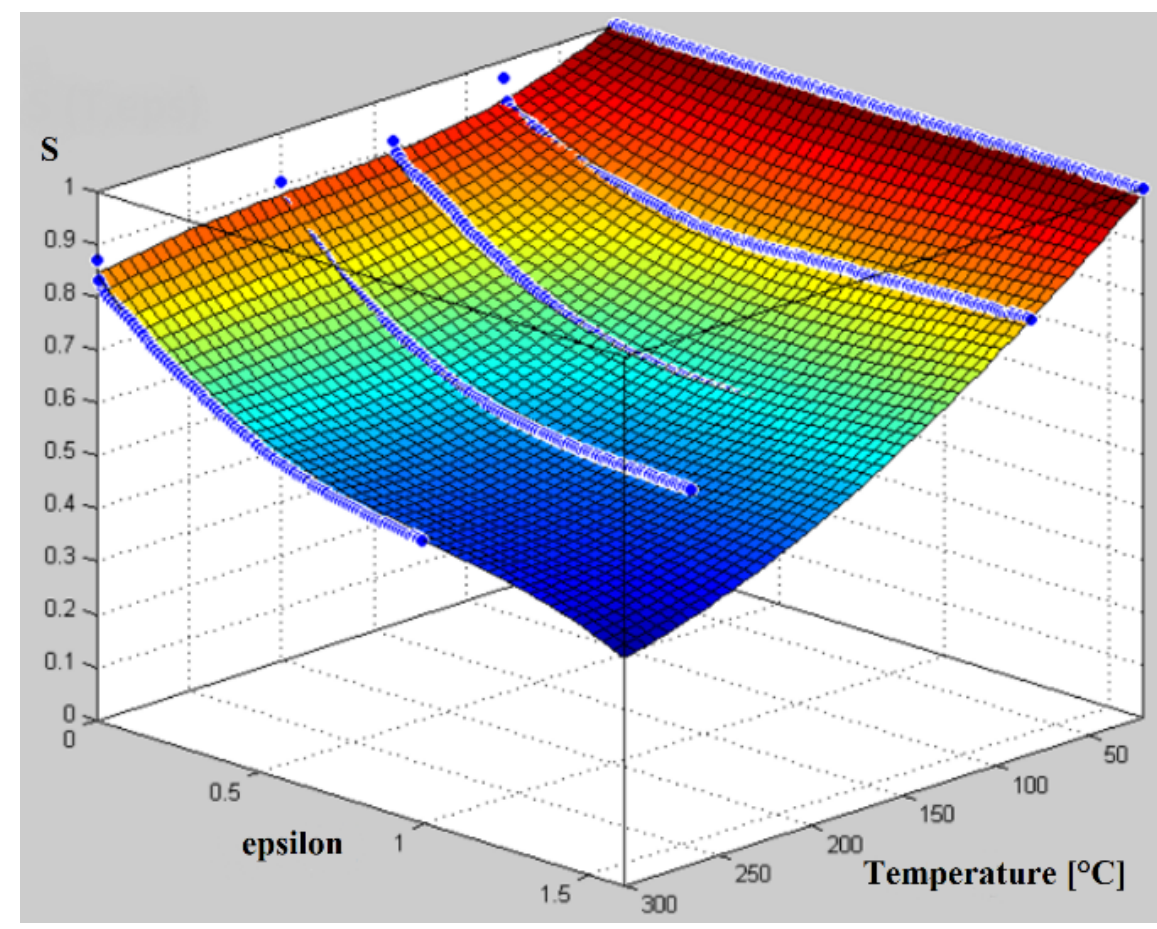

Fig. 2. 3D Thermal softening function

Assuming now a multiplicative hardening with the coupled effects of strain and temperature included within the softening function, the general Considère condition of eq. (3) must be updated; for the quasistatic case with temperature effect $\left(\frac{\partial \dot{\varepsilon}_{\text {True }}}{\partial \varepsilon_{\text {True }}}=0, \dot{\varepsilon}_{\text {True }}=0\right.$ and $\left.R=1\right)$ it takes the following form:

$\sigma_{E q-S} \bullet S-\frac{\partial \sigma_{E q-S}}{\partial \varepsilon_{\text {True }}} \bullet S-\sigma_{E q-S} \bullet \frac{\partial S}{\partial T} \bullet \frac{\partial T}{\partial \varepsilon_{\text {True }}}-\sigma_{E q-S} \bullet \frac{\partial S}{\partial \varepsilon_{\text {True }}}=0 \rightarrow \varepsilon_{N-D T}(9)$

then eq. (5) only valid if $S=S(T)$, is substituted by the more realistic eq. (10) which is based on $S=S(T, \varepsilon)$.

$\varepsilon_{N-T}=\frac{n}{1-\frac{1}{S} \cdot\left(\frac{\partial S}{\partial \varepsilon \text { True }}+\frac{\partial S}{\partial T} \cdot \frac{\partial T}{\partial \varepsilon_{\text {True }}}\right)}(10)$

The significant improvement of eq. (10) with respect to eq. (5) is that, thanks to the incorporation of the strain-temperature coupling within $S(T, \varepsilon)$ and to the related term $\frac{\partial S}{\partial \varepsilon_{\text {True }}}$, the former equation recognizes the necking onset anticipation due to constant temperatures higher than $T_{\text {Room }}$, which eq.(5) was not able to capture. 
The obtained quasi-static thermal softening is now applied to the dynamic tests, for also predicting the corresponding changes of the necking strain. The anticipation effect due to the temperature will be in contrast to the delay effect caused by the strain rate variation, and the analysis of dynamic experiments will show which one of the two effects will prevail.

Analysis of the necking phenomenon in dynamic tests with combined strain rate and temperature effects

During dynamic SHTB tests, both temperature and strain rate undergo significant variations. In fact the temperature greatly increases due to the plastic work generated in almost adiabatic conditions, while the plastic strain rate evolves from zero at first yield up to the regime value at plateau.

Then, also the necking inception strain can remarkably change with respect to $\varepsilon_{N}$ from static tests at $T_{\text {Room }}$, because of the combined effect from the thermal softening and from the dynamic amplification of the stress.

Such combined effect is very clear in the left part of Fig. 3, where the dynamic true curves are compared to the quasi-static ones at room temperature.
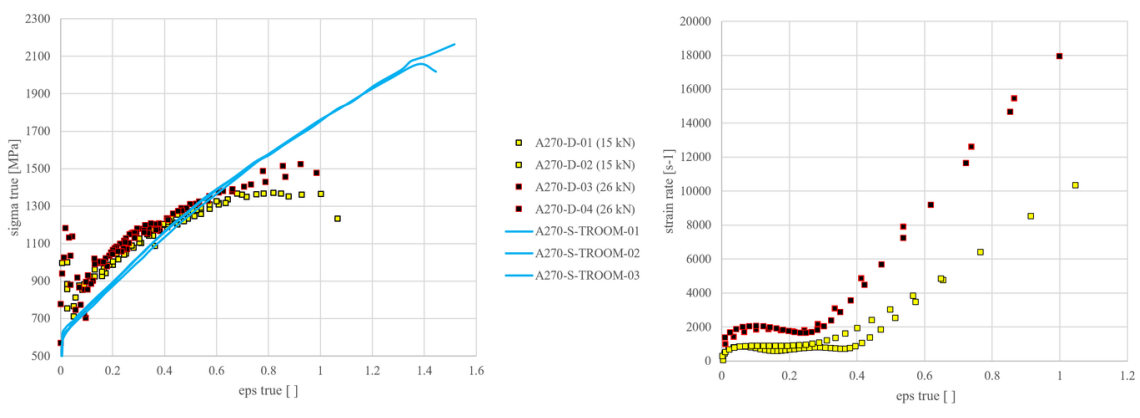

Fig. 3. True Stress-True strain curves from SHTB experiments compared to the quasi-static one at room temperature (left) and dynamic effective diameter-based true strain rate vs. true strain curves (right)

In the first part of the dynamic tests, the temperature is very close to the room one and it is possible to see a clear strain rate effect, with both dynamic curves higher than the static ones and, among the dynamics, the $26 \mathrm{kN}$ curves higher than the $15 \mathrm{kN}$ ones. Then, the temperature rises with the strain and, at the end of all the dynamic tests, it is so high that the corresponding thermal softening has a greater effect than the strain rate amplification; in fact, at late strains the dynamic true curves become lower than the quasi-static ones at room temperature.

To complete the description of the dynamic tests, in the right part of Fig. 3 also the strain rate vs strain curves are shown for all the dynamic tests.

Classical strain rate histories from SHTB tests are supposed to remain constant from the end of the rise time up to failure. Instead optical measurements of the current specimen diameter show that the necking induces an intense spontaneous increase of the effective strain rate, so that the strain rate histories in Figure 3 exhibit a plateau just limited to the necking onset, followed by a steep increasing ramp extending up to failure.

Such intense spontaneous increase of the strain rate after necking onset was firstly evidenced by Mirone ${ }^{20}$, confirmed by Mirone et al. ${ }^{21}$ and recently acknowledged by Zhang et al. ${ }^{22}$.

In order to calculate the temperature increase due to plastic work conversion during dynamic tests, it is necessary to evaluate the equivalent stress-strain curves; these are obtained here by correcting the experimental true curves all over their postnecking range, through the MLR function ${ }^{15}$. The plastic work is then converted into heat via the Taylor-Quinney Coefficient (TQC) assumed to be equal to 1, according to the findings of Kapoor \& Nemat-Nasser ${ }^{10}$ and Walley et al. ${ }^{11}$. The calculated temperature histories for all the 
dynamic tests are shown in Fig. 4. The necking strains identified from experiments are then reported in Table 3 together with the corresponding temperature at that instant.

Fig. 5 shows the necking strains against the temperature from quasi-static and dynamic tests. The dynamic necking strains (around 0.2 at nearly $70{ }^{\circ} \mathrm{C}$ ) lie below the fitting curve of the quasi-static necking strains, delivering a value of about 0.35 at $70{ }^{\circ} \mathrm{C}$.

This means that the anticipation effect induced by $\frac{\partial S}{\partial T} \bullet \frac{\partial T}{\partial \varepsilon_{\text {True }}}$ in eq.(8) is greater than the delaying effect of the strain rate, resulting in lower dynamic necking strains with respect to the quasi-static counterparts at the same temperature.

It is important to underline that, without the coupling between strain and temperature within the thermal softening function, no explanation could have been provided for the dynamic necking strains being lower than their quasi-static counterparts at the same temperature.

Fig. 4. Material temperature histories in the dynamic test Fig. 5. Quasi-static and dynamic necking inception strains

Table 3. Dynamic necking strain and corresponding temperature

\begin{tabular}{lllll}
\hline & D-01 (15 kN) & D-02 $(15 \mathrm{kN})$ & D-03 $(26 \mathrm{kN})$ & D-04 $(26 \mathrm{kN})$ \\
\hline$\varepsilon_{N-D}$ & 0.24 & 0.24 & 0.19 & 0.23 \\
$T_{N}$ & 75 & 76 & 65 & 75 \\
\hline
\end{tabular}

It is highly expectable that a coupling of strain rate and temperature also occurs under dynamic conditions, although its determination requires further tests not available for this work. However, the overall predominance of the temperature effects on the dynamic ones in affecting the necking inception is ascertained here independently of the possible further coupling above.

Failure strains under combined strain rate and temperature effects

Failure is a typically local phenomenon, so the local diameter-based true strains at fracture should be much more appropriate than the elongation-based engineering strains frequently adopted in the literature, which only reflect a global volume-averaged strain indicator.

Both types of fracture strains are reported in Table 4 for each test family (average of two or three test repetitions), together with the corresponding values of nominal strain rates and of estimated specimen temperatures at fracture. In the same table are reported also the effective / engineering strains ratio and the max postnecking true strain (Fail - Neck).

Table 4 Strain-related variables at fracture

\begin{tabular}{llllllll}
\hline & S-T20 & S-T80 & S-T140 & S-T200 & S-T300 & D 15 kN & D 26 kN \\
\hline Reference true strain rate & 0.003 & 0.003 & 0.003 & 0.003 & 0.003 & 800 & 1800 \\
Temperature at fracture & 20 & 80 & 140 & 200 & 300 & 324 & 299 \\
Fracture true strain & 1.48 & 1.53 & 1.32 & 1.27 & 1.06 & 1.07 & 0.93 \\
Fracture engineering strain & 0.67 & 0.66 & 0.45 & 0.35 & 0.30 & 0.45 & 0.35 \\
Effective / Engineering strains ratio & 1.27 & 2.32 & 2.93 & 3.63 & 3.53 & 2.38 & 2.66 \\
Max Postnecking true strain (Fail - Neck) & 1.04 & 1.18 & 1.13 & 1.09 & 0.88 & 0.83 & 0.715 \\
\hline
\end{tabular}

The same data are arranged in Fig. 6 as a 3D plot with the reference true strain rate and the temperature at fracture as the $\mathrm{X}$ and $\mathrm{Y}$ axes and the true strain at fracture as the $\mathrm{Z}$ axis. 


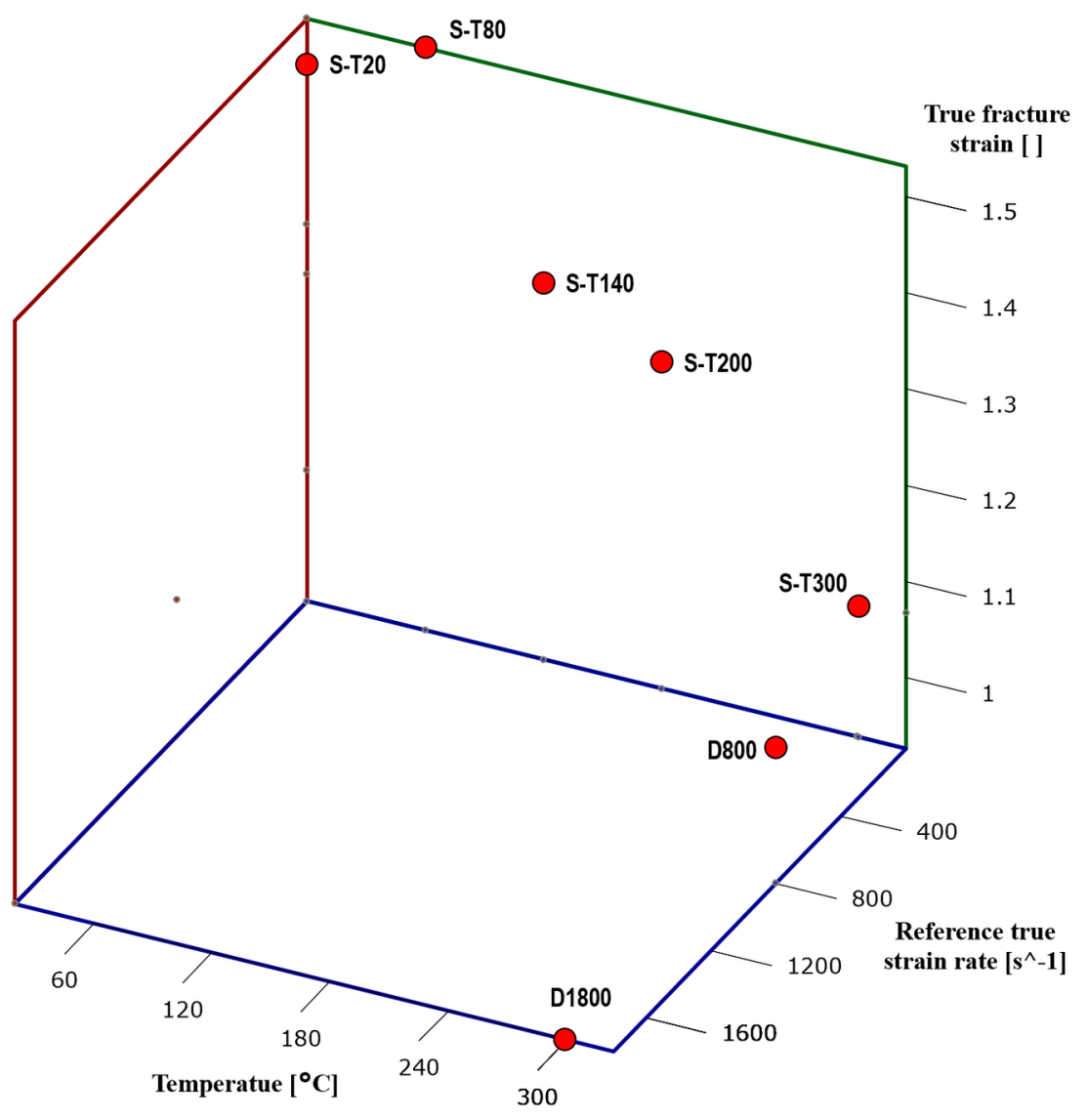

Fig. $63 \mathrm{D}$ plot of the true fracture strain versus temperature at fracture and reference true strain rate of the test

The fracture true strain at static rates clearly decreases with the temperature, from 1.48 at room temperature to 1.06 at $300{ }^{\circ} \mathrm{C}$. This can also be indirectly caused by the temperature anticipating the necking onset, which, in turn, causes the necking-induced stress triaxialty to evolve much sooner and much largely than it does for tests at room temperature. In fact, the postnecking strain range up to failure is nearly identical for static tests from Troom up to $200{ }^{\circ} \mathrm{C}$ and slowly decreases at $300{ }^{\circ} \mathrm{C}$.

Dynamic tests exhibit a material temperature at fracture close to $300{ }^{\circ} \mathrm{C}$, similar to the quasi-static tests at the highest temperature, but the true strains at failure are lower, close to 0.93 for the tests at nominal strain rate of $1800 \mathrm{~s}^{-1}$. This means that the strain rate too tends to anticipate failure of the A270 steel together with temperature, by further decreasing the fracture strain.

The dynamic tests at nominal $800 \mathrm{~s}^{-1}$, progressively heating from Troom up to $300{ }^{\circ} \mathrm{C}$ at incipient failure, exhibits a failure strain close to that of static $300{ }^{\circ} \mathrm{C}$ : the fracture-delaying effect of the initially low temperature of the dynamic test with respect to the static one (at $300{ }^{\circ} \mathrm{C}$ since first yield) is compensated by the fracture-anticipating strain rate effect. Therefore, for the A2-70 steel at hand, both temperature and strain rate have a decreasing effect on the fracture true strain.

Comparing the necked specimen shapes from dynamic tests to those from the quasi-static tests at $300{ }^{\circ} \mathrm{C}$, it is also possible to see that they show different degrees of strain localization at fracture, fully reflecting the maximum postnecking strain in the last row of Table 4. 
Fig. 7 shows the comparison between the last frames before fracture of the S-T300 and the D-26, where the difference between the two diameters is highlighted. The two tests series show a similar fracture engineering strain (0.3 and 0.35 respectively) but different fracture true strain (1.06 and 0.93 respectively), i.e. different diameter. In other words, the test S-T300 shows a greater strain localization than the dynamic test at 1800 $\mathrm{s}^{-1}$, despite a comparable overall engineering deformation.

Similarly, in Fig. 8 it is shown the comparison between the last frames before fracture of the S-T300 and the D-15, in which is highlighted the difference between the two gage lengths. In this case, the two group of tests show a similar fracture true strain (1.06 and 1.07 respectively), i.e. similar diameter, but different fracture engineering strain (0.3 and 0.45 respectively), i.e. different overall gage length. Therefore, the S-T300 shows a similar fracture true strain in respect to the D-15 with a lower overall engineering deformation.

Both comparisons highlight that the high temperature quasi-static tests show a greater strain localization with respect to the dynamic tests. This is due to the earlier necking onset of static high temperature tests with respect to the dynamic tests at room temperature, leading to larger postnecking strains at the same overall true strain which either means more pronounced shrinking at given elongation or lower elongation at given diameter contraction.

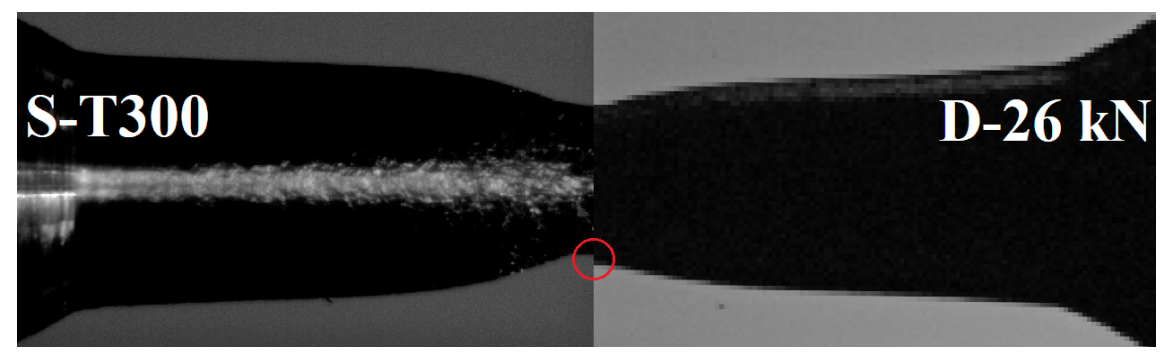

Fig. 7 Comparison at fracture between S-T300 and D-26 kN with highlighted the difference between the diameters, i.e. between the true strains, with a similar gage length, i.e. similar engineer strain

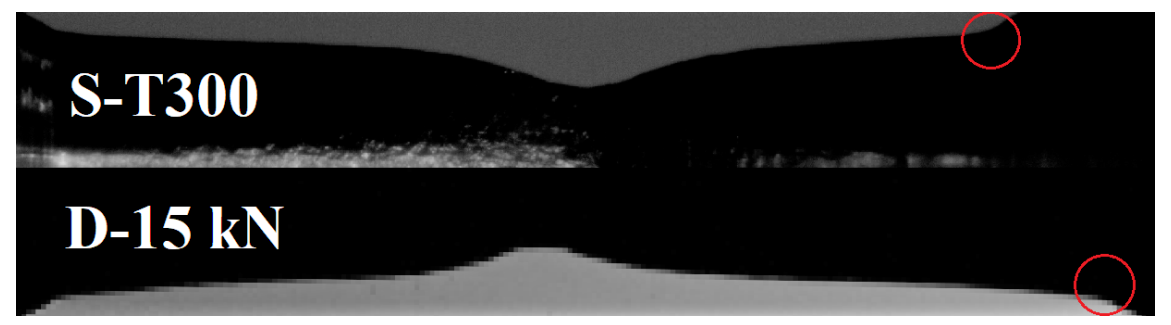

Fig. 8 Comparison at fracture between S-T300 and D-15 kN with highlighted the difference between the gage lengths, i.e. between the engineering strains, with a similar diameter, i.e. similar true strain

\section{Conclusions}

In the present work, an in-depth analysis of the necking onset under quasi-static and dynamic conditions has been carried out for the A2-70 stainless steel.

Firstly the influences of temperature and strain rate together with their variability have been evaluated on the necking inception strain, by assuming that strain, strain rate and temperature are uncoupled to each other. From such qualitative analysis it was shown that, in standard SHTB tests, the increasing temperature and strain rate should respectively cause a decrease and an increase of the necking initiation strain.

The experimental campaign on the A2-70 steel included quasi-static tests at different temperatures and dynamic SHTB test at room temperatures, all with cylindrical specimens. The true stress - true strain 
curves, derived from the tests by means of fast camera acquisitions of the evolving diameter, are translated into equivalent stress-strain curves by using the MLR function.

The necking anticipation found from quasi-static experiments at constant high temperatures demonstrated the existence of a coupling between strain and temperature within the thermal softening function. This necking anticipation was then mathematically demonstrated by simply introducing a general coupling between strain and temperature within the thermal softening function.

The evolving temperatures of all the tests, due to the fast adiabatic conversion of plastic work into heat, were evaluated; then, the triplets of strain, temperature and thermal softening values were best-fitted by a general polynomial.

The dynamic necking strains lower than their quasi-static counterparts at similar temperatures evidenced that the anticipating effect caused by the growing temperature is greater than the delaying effect of the growing strain rate.

Lastly, the analysis of fracture strains showed that, for the A2-70 steel at hand, both temperature and strain rate have a decreasing effect on the fracture true strain. Moreover, comparing the necked specimens from dynamic tests to those from quasi-static tests at high temperature, an influence of temperature on the strain localization and on the maximum postnecking strain range was evidenced and addressed to earlier necking strains from higher temperatures.

Author contribution statement

All persons who meet authorship criteria are listed as authors, and all authors certify that they have participated sufficiently in the work to take public responsibility for the content, including participation in the concept, design, analysis, writing, or revision of the manuscript.

\section{References}

[1] Ruggiero, Andrew, et al. Strain rate effects on fracture behavior of austempered ductile irons. In: AIP Conference Proceedings. AIP Publishing LLC, 2018. p. 070028.

[2] Scapin, M.; Peroni, L.; Fichera, C. Investigation of dynamic behaviour of copper at high temperature. Materials at High Temperatures, 2014, 31.2: 131-140.

[3] Sasso, Marco, et al. High strain rate behaviour of AA7075 aluminum alloy at different initial temper states. In: Key Engineering Materials. Trans Tech Publications Ltd, 2015. p. 114-119.

[4] Mirone, G., et al. Static and dynamic response of titanium alloy produced by electron beam melting. Procedia Structural Integrity, 2016, 2: 2355-2366.

[5] Mirone, G.; Barbagallo, R.; Giudice, F. Locking of the strain rate effect in Hopkinson bar testing of a mild steel. International Journal of Impact Engineering, 2019, 130: 97-112.

[6] Hart, E. W. Theory of the tensile test. Acta metallurgica, 1967, 15.2: 351-355.

[7] Ghosh, A. K. Tensile instability and necking in materials with strain hardening and strain-rate hardening. Acta Metallurgica, 1977, 25.12: 1413-1424.

[8] Guan, Zhiping. Quantitative analysis on the onset of necking in rate-dependent tension. Materials \& Design (1980-2015), 2014, 56: 209-218.

[9] Lin, E. I. H. Plastic instability criteria for necking of bars and ballooning of tubes. 1977.

[10] Kapoor, Rajeev; Nemat-Nasser, Sia. Determination of temperature rise during high strain rate deformation. Mechanics of Materials, 1998, 27.1: 1-12.

[11] Walley, S. M., et al. Comparison of two methods of measuring the rapid temperature rises in split Hopkinson bar specimens. Review of scientific instruments, 2000, 71.4: 1766-1771. 
[12] Jovic, C., et al. Mechanical behaviour and temperature measurement during dynamic deformation on split Hopkinson bar of 304L stainless steel and 5754 aluminium alloy. In: Journal de Physique IV (Proceedings). EDP sciences, 2006. p. 1279-1285.

[13] Rittel, D.; Zhang, L. H.; Osovski, S. The dependence of the Taylor-Quinney coefficient on the dynamic loading mode. Journal of the Mechanics and Physics of Solids, 2017, 107: 96-114.

[14] Bridgman, Percy Williams. Studies in large plastic flow and fracture. New York: McGraw-Hill, 1952.

[15] Mirone, Giuseppe. Approximate model of the necking behaviour and application to the void growth prediction. International Journal of Damage Mechanics, 2004, 13.3: 241-261.

[16] Mirone, G.; Verleysen, Patricia; Barbagallo, R. Tensile testing of metals: Relationship between macroscopic engineering data and hardening variables at the semi-local scale. International Journal of Mechanical Sciences, 2019, 150: 154-167.

[17] Peroni, Lorenzo; Scapin, Martina; Fichera, Claudio. An advanced identification procedure for material model parameters based on image analysis. In: 10th European LS-DYNA Conference tenutosi a Würzburg (Germania). 2015.

[18] Sasso, M., et al. High speed imaging for material parameters calibration at high strain rate. The European Physical Journal Special Topics, 2016, 225.2: 295-309.

[19] Ashuach Y., Avinadav C., and Rosenberg Z. Eliminating the thermal softening of dynamically loaded specimens in the Kolsky bar system by multi-step loading, EPJ Web of Conferences, 2012, 26, 01047.

[20] Mirone, Giuseppe. The dynamic effect of necking in Hopkinson bar tension tests. Mechanics of materials, 2013, 58: 84-96.

[21] Mirone, G.; Corallo, D.; Barbagallo, R. Experimental issues in tensile Hopkinson bar testing and a model of dynamic hardening. International Journal of Impact Engineering, 2017, 103: 180-194.

[22] Zhang, Longhui, et al. Rate dependent behaviour and dynamic strain localisation of three novel impact resilient titanium alloys: Experiments and modelling. Materials Science and Engineering: A, 2020, 771: 138552. 